\title{
Role of stress, age and adjuvant therapy in the cognitive function of patients with breast cancer (Review)
}

\author{
ARTEMIS PAPANASTASIOU ${ }^{*}$, THEODORA SELINIOTAKI ${ }^{2 *}$, EMMANOUIL RIZOS $^{1}$, KATERINA KAMPOLI $^{2}$, \\ ANASTASIOS NTAVATZIKOS ${ }^{2}$, NIKOLAOS ARKADOPOULOS ${ }^{3}$, CHRISTINA TSIONOU $^{4}$, \\ DEMETRIOS A. SPANDIDOS ${ }^{5}$ and ANNA KOUMARIANOU ${ }^{2}$ \\ ${ }^{1}$ Second Department of Psychiatry, ${ }^{2}$ Hematology-Oncology Unit, Fourth Department of Internal Medicine, and \\ ${ }^{3}$ Fourth Department of Surgery, University General Hospital 'ATTIKON', School of Medicine, \\ National and Kapodistrian University of Athens, 12462 Athens; ${ }^{4}$ Department of Breast Diseases, \\ Maternity-Health, 15232 Athens; ${ }^{5}$ Laboratory of Clinical Virology, Medical School, \\ University of Crete, 71003 Heraklion, Greece
}

Received March 29, 2019; Accepted May 10, 2019

DOI: $10.3892 / \mathrm{ol} .2019 .10361$

\begin{abstract}
According to data largely obtained from retrospective studies, it has been postulated that chemotherapy exerts an aggravating effect on the cognitive function of patients with breast cancer. Potential individual factors related to the effects of chemotherapy on cognitive function have been indicated, such as age-related cognitive dysfunction and stress. Elderly patients differ from non-elderly patients as regards higher cognitive related comorbidities, such as dementia, as well as regarding lower stress levels, indicating that 'chemobrain' may differentially affect these two age groups. The aim of this review was to discuss the effects of stress and chemotherapy on cognitive dysfunction and identify any potential age-related differences in patients with breast cancer treated with adjuvant chemotherapy. For this purpose, a systematic review of the literature was carried out on the PubMed, Scopus and Web of Science databases. The inclusion criteria were original articles published in peer-reviewed journals, elderly and non-elderly patients with breast cancer, reporting on stress and at least one cognitive parameter pre- and/or post-treatment. Eight studies met the preset criteria and were further analyzed. In total, the data of 1,253 women were included, of whom 800 patients with breast cancer were treated with surgery only, systemic treatment only, or both. Although all the studies included a
\end{abstract}

Correspondence to: Dr Anna Koumarianou, HematologyOncology Unit, Fourth Department of Internal Medicine, University General Hospital 'ATTIKON', School of Medicine, National and Kapodistrian University of Athens, 1 Rimini Street, Chaidari, 12462 Athens, Greece

E-mail: akoumari@yahoo.com

${ }^{*}$ Contributed equally

Key words: cognitive function, stress, 'chemobrain', geriatric, chemotherapy, breast cancer non-elderly breast cancer patient subpopulation, only two of the studies included patients over 65 years of age. All studies indicated a statistically significant association of stress with various domains of cognitive dysfunction in patients, as shown by either self-completed questionnaires, neuropsychological testing or both. An age over 60 years was linked to fewer cognitive difficulties mediated by lower levels of stress. Thus, the evidence supports the association of stress with cognitive deficits in patients with breast cancer, regardless of the type of cancer-related treatment. Therefore, stress should be appropriately addressed. However, further research is required to investigate the association of stress with cognitive function in elderly patients with breast cancer.

\section{Contents}

1. Introduction

2. Data collection methods

3. Interpretation of the collected data

4. Involvement of stress, age and adjuvant therapy on cognitive function of patients with breast cancer

5. Limitations to the data interpretation

\section{Introduction}

Breast cancer is the leading type of cancer affecting females worldwide and accounts for $25 \%$ of cancer incidence among women $(1,2)$. Epidemiological analyses from the Surveillance, Epidemiology, and End Results Program (SEER) database in the USA have indicated that the majority (almost 60\%) of breast cancer cases are diagnosed at 65 years or older and $>30 \%$ of the cases are diagnosed over the age of 70 , thus rendering breast cancer the leading cause of cancer-related mortality among women older than 65 years of age (3).

Surgery, radiotherapy and systemic therapy, including chemotherapy and endocrine therapy, are the most common 
treatment methods for patients with breast cancer. However, the administration of systemic therapy has been shown to be associated with cognitive dysfunction described as 'chemobrain' or chemotherapy-related cognitive dysfunction $(4,5)$. 'Chemobrain' refers to limitations in memory, concentration, learning ability, processing speed, language and executive function (4,6). Although the majority of studies describe cognitive dysfunction related to chemotherapy as a reversible side-effect that usually occurs within 18 to 36 months following diagnosis, a large retrospective analysis from the SEER database including 18,360 patients indicated a long-term increased risk of dementia $(5,7)$.

Stress, as a consequence of significant environmental factors, has also been shown to be associated with poorer cognitive function and has been shown to exert a damaging effect on brain regions involved in cognitive performance, such as the prefrontal cortex and the hippocampus (8). Prolonged exposure to stress can accelerate the aging process and increase the risk of developing dementia and other cognitive deficits $(9,10)$. Patients with breast cancer have to cope with several types of stress during their everyday life as a result of the disease itself and the side-effects of the applied treatments (e.g., neuropathy, bone pain, hair loss, etc.) $(11,12)$. As indicated by case-control studies, patients with breast cancer undergoing active treatment have elevated stress levels compared to healthy individuals $(13,14)$. Of interest, recent evidence indicates that younger patients experience higher stress levels as compared to older ones (15).

Moreover, it is well known that normal aging affects a wide range of cognitive domains, such as memory, processing speed, executive function, shift of attention, visuospatial and constructional skills (16). Structural and functional changes mediate this decline, which has been highlighted by neuropsychological and neuroimaging studies (16-20). Nevertheless, the prevalence of cognitive disorders, such as dementia, is higher among the elderly $(16,21)$. Recent advances in medicine and technology, have led to major declines in mortality, not only of younger-age patients, but also of older-age ones, resulting in a marked increase in the elderly population worldwide. Thus, it has become a great challenge to safeguard cognitive functions and to preserve independence and functionality in the aging population. Geriatric patients with breast cancer have increased supportive care needs due to age-related functionality decline, more comorbidities and a lower tolerance to treatment regimens, i.e., chemotherapy and radiotherapy (22). However, more elderly patients are expected to be treated in the future as, a recent analysis from the SEER database including 160,676 women aged 65 years and older (21,743 aged older than 80 years) indicated an overall survival benefit from chemotherapy, particularly in those assessed as 'good risk' by geriatric assessment tools (23).

The aim of this review was to shed light on the role of systemic therapies, stress, and age in the cognitive function of patients with early-stage breast cancer. In addition, this review aimed to discuss the differential effects of chemotherapy and stress on the cognitive function of elderly and younger patients with breast cancer.

\section{Data collection methods}

Study design. For the purpose of this study, we conducted a systematic review of the literature. A.P. and T.S. participated in the literature search, study selection process and extraction of the studies. Disagreements between the authors were resolved through discussion or with the assistance of A.K. All authors participated in the appraisal of the extracted studies.

Literature search. A search for articles in the English language published from database inception until September 23, 2018 was carried out on the PubMed, Scopus and Web of Science databases. The combinations of keywords used were as follows: ('breast cancer' OR 'breast carcin*' OR 'breast tumor*') AND (cognit* OR 'executive function' OR attention OR memory OR orientation OR perception OR language OR verbal OR visual $\mathrm{OR}$ vigilance $\mathrm{OR}$ visuospatial $\mathrm{OR}$ 'problem solving' OR 'processing speed') AND chemotherapy AND (stress OR stressor OR cortisol OR 'perceived stress'). A second search was also carried out using the terms ('breast cancer' OR 'breast carcin*' OR 'breast tumor*') AND (cognit* OR 'executive function' OR attention OR memory OR orientation OR perception $O R$ language $O R$ verbal $O R$ visual $O R$ vigilance OR visuospatial OR 'problem solving' OR 'processing speed') AND chemotherapy AND (toxicity OR side-effect* OR 'adverse event*' OR chemobrain OR chemofog OR 'adverse react*' OR 'central nervous system'). In addition, a snowball technique was utilized in order to include any potential studies not revealed through this process. Issues of related journals, reference lists of included studies, and other relevant articles in the field were rummaged in an attempt to locate possible records. The flow of information from record identification to inclusion followed the principles of the Preferred Reporting Items for Systematic review and Meta-Analysis Protocols statement (PRISMA) (24) and is presented in Fig. 1.

Study selection. The inclusion criteria were as follows: i) Original articles published in peer-reviewed journals; ii) studies including elderly and/or non-elderly patients with breast cancer; and iii) studies assessing stress and cognitive function of such patients pre- and/or post-treatment. The exclusion criteria were the following: i) Preclinical and interventional type of studies; and ii) diagnosis of brain metastasis.

Extracted data. The extracted data of these articles included the following: Name of study, country, total number of patients, total number of participants, sample characteristics, measures of stress, measures of cognitive function, timing of assessments, and association of stress with cognitive parameters (Table I).

Appraisal of the quality of the studies. The quality of the included studies was evaluated using the Newcastle-Ottawa Scale proposed by the Cochrane Non-Randomized Studies Methods Working Group (25). This instrument was selected as it can be applied to evaluate studies involving different methodological study designs in a single assessment process. In particular, as regards the assessment of case-control studies, the Newcastle-Ottawa Scale includes 8 items which control for the selection of the cases and controls (adequate definition and representativeness of the cases, selection and definition of controls), the comparability between cases and controls with respect to certain factors (i.e., age, sex, etc.) and the degree of exposure (method of ascertainment of exposure, similarity of 


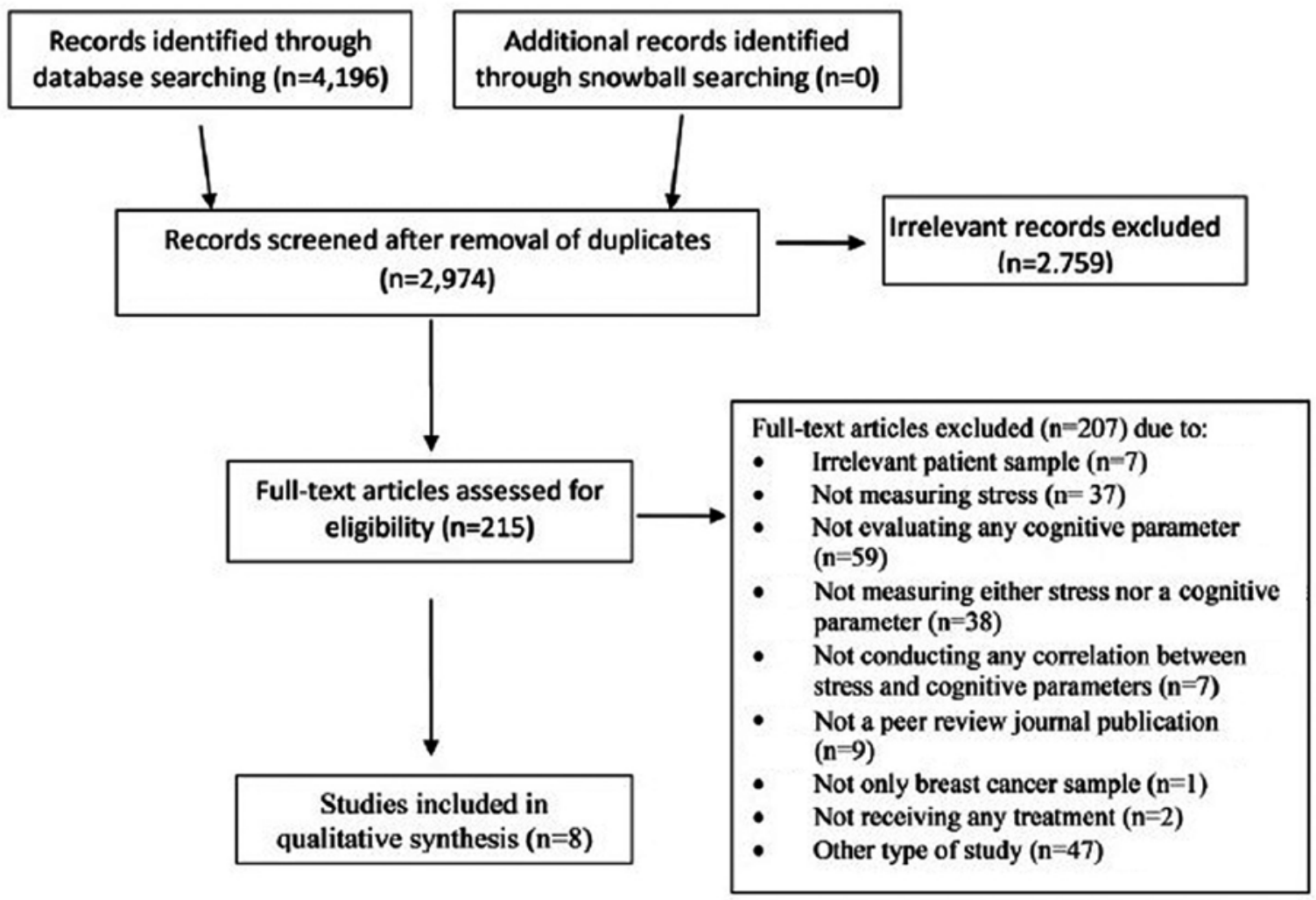

Figure 1. The flow of information from record identification to inclusion used in this review.

method for cases and controls, non-response rates for groups). A maximum of 4 stars can be given for the selection subscale, 2 for the comparability subscale and 3 for the exposure subscale, summing up to a maximum of 9 stars (Table II).

\section{Interpretation of the collected data}

Of the 4,196 study records retrieved through database searching, 8 met the pre-set eligibility criteria and were included in this review (Fig. 1) (26-33). Of these, 6 were case-control studies (26-30,33) and 2 that had no control group used literature data for comparative analysis $(31,32)$. Only 2 of the studies were longitudinal and assessed the participants both before and at standard times following the completion of chemotherapy (26,33). From the point of view of age, 2 studies included patients under and over 65 years of age $(26,30)$, but only one analyzed them as a separate group (30). With respect to systemic treatment, 1 study examined patients just prior to the initiation of adjuvant treatment (29), 2 studies included patients on active chemotherapy $(26,33)$ and 5 studies described patients who had completed chemotherapy from 2 weeks up to 10 years earlier $(27,28,30-32)$. Two studies included groups of patients who had only received surgery at the time of the assessment, either prior to the initiation of chemotherapy or not requiring further treatment $(26,33)$. In all the studies, the study participants could have received additional types of treatment (i.e., endocrine treatment and radiotherapy), apart from surgery and chemotherapy

In total, 1,253 women were included in the analysis. Different aspects of stress were investigated in different studies, namely generalized stress $(26,29-31)$, job-related stress (28), trauma-related stress $(32,33)$ and physiological response to stress (27). Accordingly, stress assessment methods varied among the studies. Five studies used validated questionnaires (26,29-32). One study used a single item of a battery to assess the responders' job-related stress (28). One study used a structured clinical interview for post-traumatic stress disorder symptoms (33) and 2 studies measured cortisol levels during the day and under a laboratory controlled task respectively $(27,32)$. The fields of cognitive function examined in the 8 studies were attention $(26,28,29,33)$ cognitive flexibility $(28,29)$ executive function $(26,28,29,33)$ concentration $(29,30,33)$ verbal and visual memory $(26,28,29,33)$ episodic memory (29), working memory $(27,28)$, verbal paired associated memory $(27)$ processing speed $(26,29,33)$ functional and verbal tasks $(26,33)$ stress-enhanced recall $(27)$, general intelligence (29), perceived cognitive impairments, comments from others, perceived cognitive abilities, impact on quality of life $(31,32)$ and work output $(28)$.

All the studies demonstrated a statistically significant association of stress with at least one cognitive parameter in patients with breast cancer. One study reported a significant association of perceived cognitive deficits with psychological stress in chemotherapy-treated patients, although a control group was not included (31). However, similar results were reported from a study comparing non-chemotherapy-treated patients with a healthy control group (29). Specifically, a worse perceived cognitive function associated with psychological distress in patients was also highlighted in a study comparing non-chemotherapy-treated patients with a healthy control group (29) despite the absence of differences between patients and controls in the neuropsychological testing. 


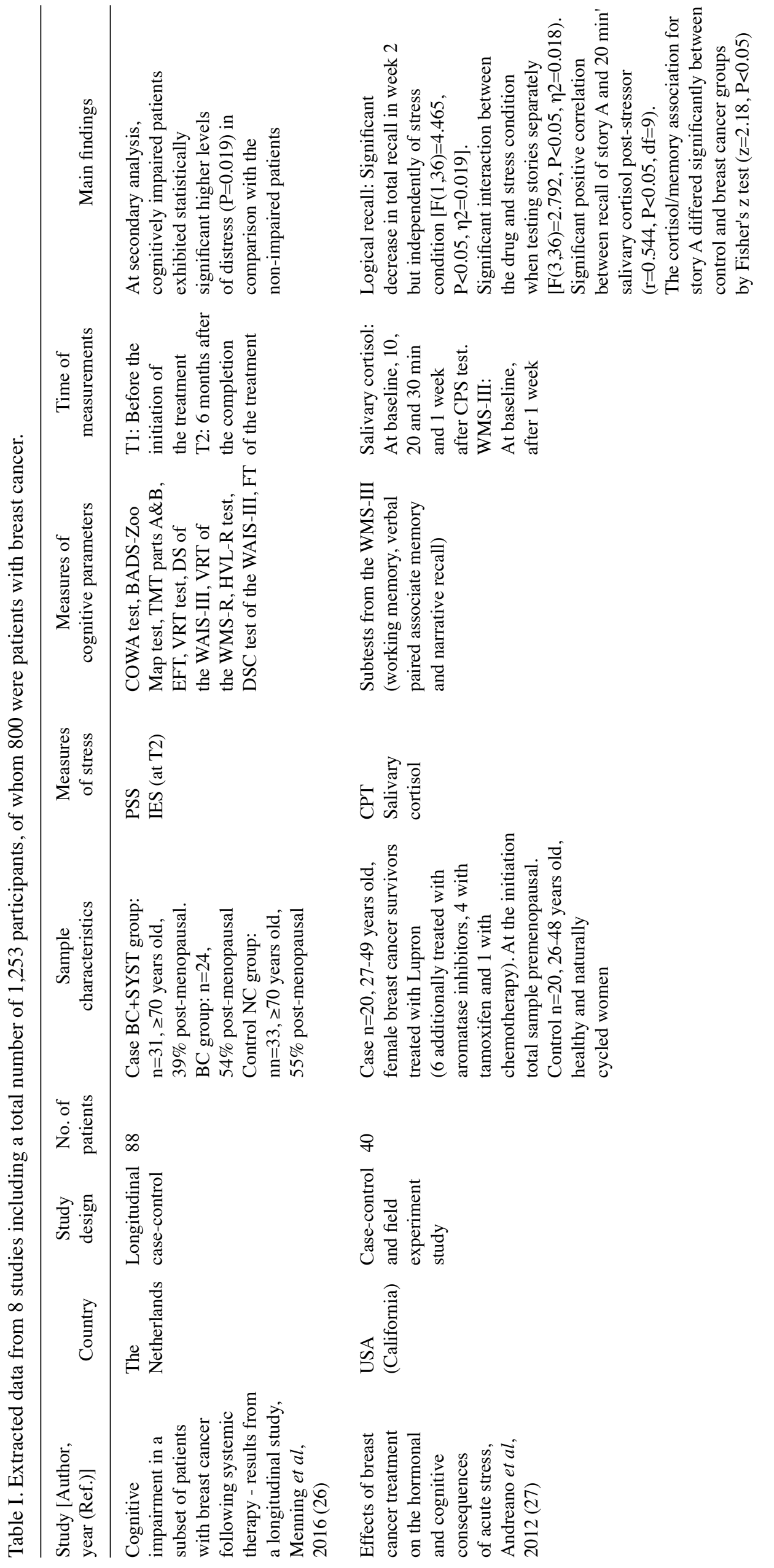

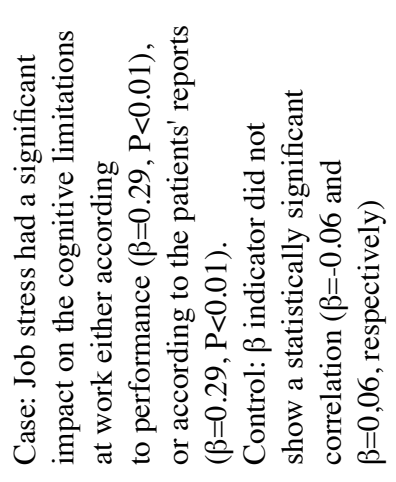

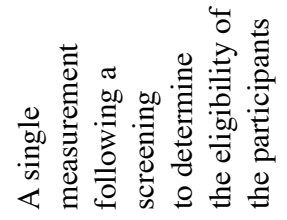

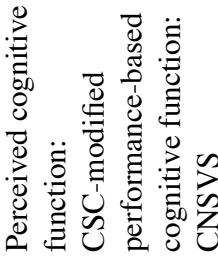

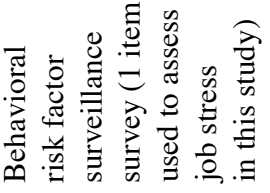
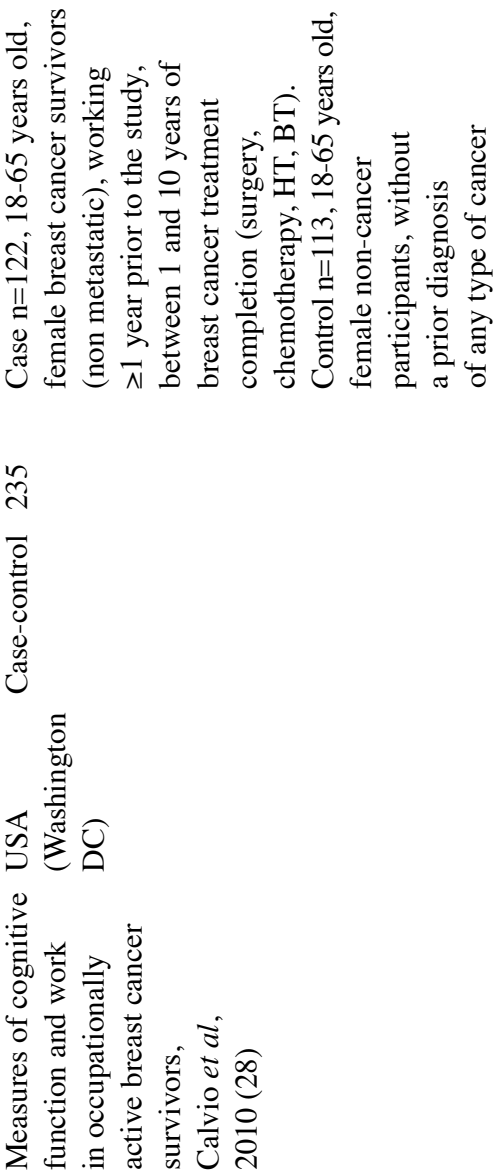


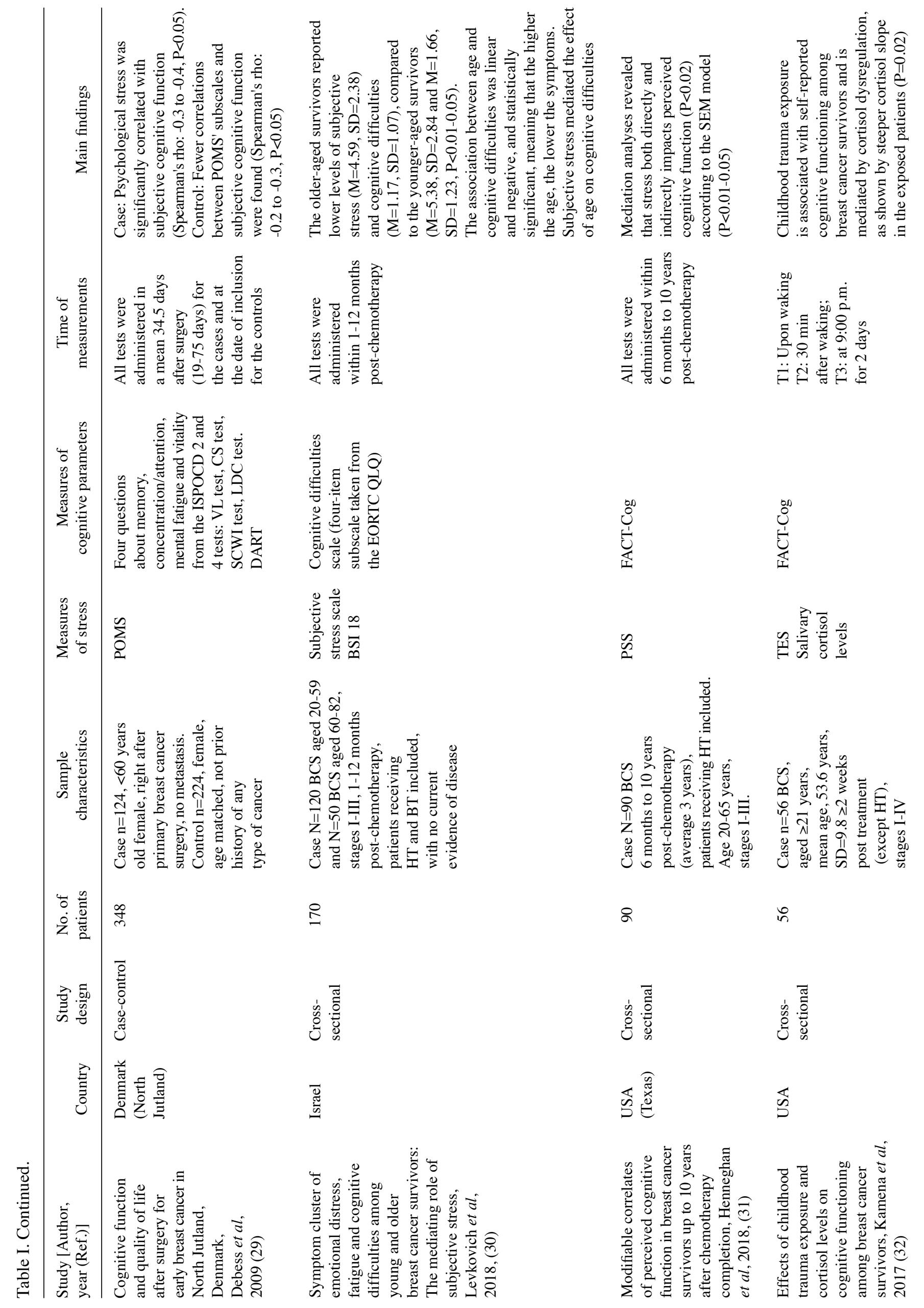




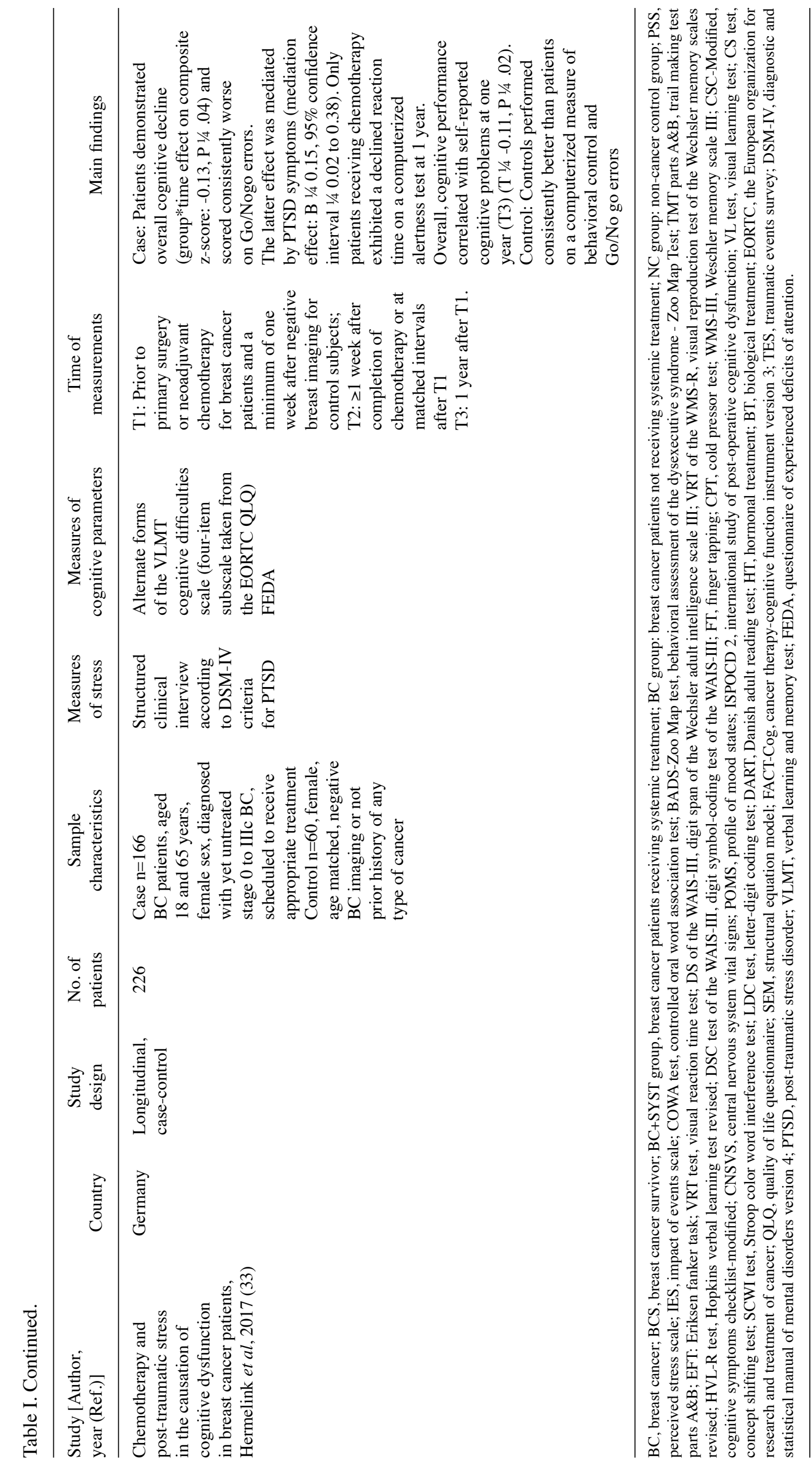


Moreover, Menning et al demonstrated that patients with cognitive dysfunction experienced higher levels of anxiety compared to the non-impaired, irrespectively of whether they were receiving or adjuvant therapy not (26). Specifically, the authors found that impaired patients exhibited a worse physical $(\mathrm{P}=0.002)$ and social functioning $(\mathrm{P}=0.004)$, and more symptoms of anxiety and depression $(\mathrm{P}=0.008)$ at 6 months post-chemotherapy, compared to the non-impaired patients. With respect to job-related stress, this was found to be significantly associated with both objective and subjective cognitive limitations at work, compared to the controls (28). In a study with an experimental stressor task, the patients with breast cancer exhibited neither cortisol release nor memory enhancement in response to the stressor, unlike the healthy controls (27). Two studies looking into the trauma-related stress found a stress-mediated association between cognitive dysfunction in chemotherapy-treated breast cancer patients, as reported in both subjective $(32,33)$ and objective cognitive assessments (33). As regards older-aged patients treated with chemotherapy, it was demonstrated that lower levels of subjective stress mediated the inverse association of age with self-rated cognitive deficits, as opposed to younger-aged patients (30).

As for the quality appraisal of the included studies, it was found to be modest as for the selection subscale, perfect as for the comparability scale, and insufficient as for the exposure subscale. The total scoring of the Newcastle-Ottawa Scale for the eight studies ranged from 4 to 8 stars. A detailed display of the qualitative analysis of the eligible studies of this systematic review according to the criteria of the Newcastle-Ottawa Scale is presented in Table II.

\section{Involvement of stress, age and adjuvant therapy on cognitive function of patients with breast cancer}

This review aimed to search for the association between stress and cognitive function in systematically treated patients with breast cancer, bearing in mind the role of age in this association. The results obtained from our search indicated that research to date has mainly focused on non-elderly patients with the exception of one study (30). The scarcity of such results downgrades the comparability of the findings, but does not debar a critical appraisal of the results.

In total, all of the included studies revealed an association of some aspect of stress with some cognitive parameters. Both generalized stress, job stress and post-traumatic stress disorder symptoms do seem to mediate cognitive deficits in systemically treated patients, although such difficulties were reported, yet to a lesser degree, to breast cancer patients not receiving systematic treatment $(26,29,33)$. Age was found to be ass with lower stress levels in patients with breast cancer $>65$ years of age (30), which is in line with previous studies $(14,34-36)$ and the lower stress levels were in turn associated with better cognitive function, as shown in the studies included in this review.

Depression is an important confounding factor that should be considered, keeping in mind the well-known burdening effect it has on cognitive function and the increased prevalence of affective disorders in oncologic patients $(37,38)$. Only one study did not conduct an assessment of depression (27). Five studies assessed and included depression in a regression analysis/linear mixed effects model, with no significant changes of the main findings $(28,30,31-33)$. In 2 studies $(26,29)$, depression as a covariate was not associated with objective tests of cognitive function. Thus, we have grounds to believe that our results were not affected by the presence of a mood disorder.

Moreover, a factor increasing the internal validity of our findings is the use of neuropsychological assessments to evaluate cognitive function, which are considered highly reliable (39).

Future research should focus on the investigation of the stress-mediated cognitive dysfunction in elderly patients with breast cancer, since as indicated by the results of this systematic review, only studies for the non-elderly population were located. Particular emphasis should be placed on the cognitive domains that tend to decline with age, i.e., memory, processing speed, etc., as opposed to those which remain relatively intact, such as language skills. Studies have shown that cognitive function is impaired for at least 2 years following diagnosis and treatment in elderly patients, whereas follow-up measurements in studies with younger patients have not shown such a maintained effect. A large longitudinal Chinese study of 1,300 chemotherapy-treated patients with breast cancer between the ages of 20 and 75 demonstrated significant cognitive improvement at the 18 and 36 months of follow-up; however, this improvement was significantly lower for older patients (4). Likewise, a Canadian study of 100 patients with breast cancer with a median age of 48 years revealed a significant reduction in adjuvant therapy-related cognitive deficits at 1 and 2 years post-treatment (40). By contrast, cognitive dysfunction seemed to persist for at least 2 years among a tamoxifen-treated population of 179 Dutch post-menopausal patients with breast cancer with a mean age of 68 years (41). Still, a long-term dysfunction in brain areas related to cognitive function has been shown in Positron Emission Tomography studies of younger patients; however, since there are no neuroimaging studies investigating long-term cognitive function in older patients, a comparison between the two age groups is of greater difficulty $(42,43)$.

Furthermore, since cognitive dysfunction is a partially stress-mediated treatment side-effect, future research should look into a potentially beneficial effect of stress management techniques on the cognitive function of patients with breast cancer. These types of methods, i.e., progressive muscle relaxation, either on their own or as a part of combinational programs including different techniques, have been shown to decrease the psychological burden and stress levels in this patient group $(44,45)$. Therefore, clinicians should keep a low threshold for offering psychotherapy and medication treatment for stress in these patients. An integrative model of medical care, in which the various patients' needs (e.g., medical, nursing, psychosocial) are compiled, and has been shown to improve their quality of life and, possibly, clinical outcomes (46).

\section{Limitations to the data interpretation}

Despite the clinical importance of stress management in patients with breast cancer, there are several limitations to take into account as regards the interpretation of our results, particularly given the heterogeneity of the included studies. Significant differences were noted regarding treatments used 


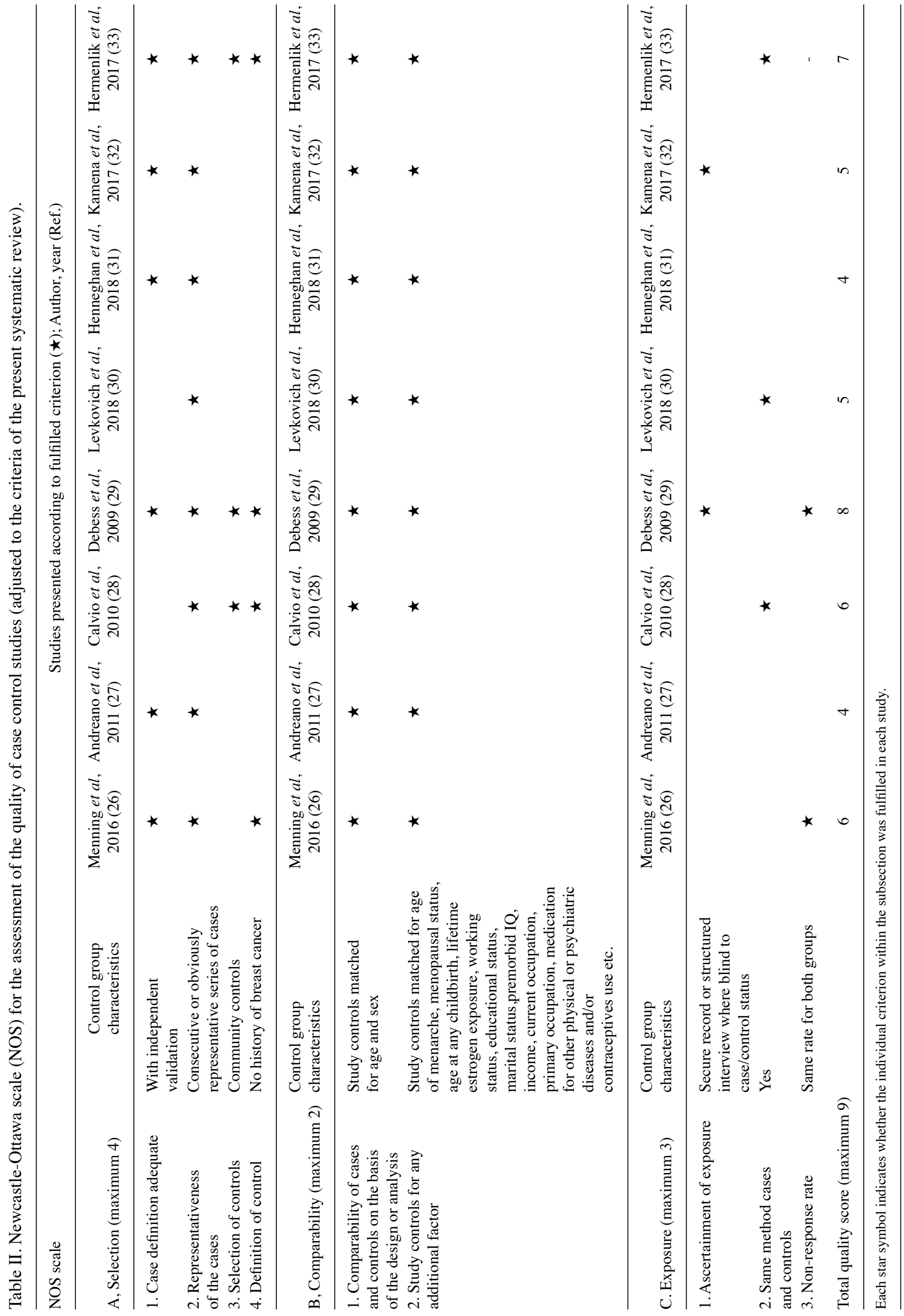


or combined (chemotherapy, endocrine treatment and radiotherapy, with or without surgery), patient traits (as per age and various demographic data, i.e., level of education) and methodologies utilized.

First, in all groups treated with chemotherapy, patients receiving other types of systematic treatment (i.e., endocrine therapy \pm radiotherapy) were not excluded; therefore, any outcome on cognition cannot be solely interpreted as a direct effect of chemotherapy. It is well known that beyond chemotherapy, endocrine therapy with or without radiotherapy have also been linked to the impairment of the cognitive function of patients with breast cancer (47-49). Still, the majority of patients, in the publications included in this review, were either under chemotherapy at the time of the study or had received chemotherapy from 2 weeks up to 10 years earlier had long-lasting cognitive impairment (50-52).

In addition, there may be a possible bias in the assessment of cognitive function, given that 3 of the studies (30-32) used self-rated questionnaires, whereas 4 of them used both objective and subjective methods $(26,28,29,33)$. Therefore, recall bias and an 'over-alertness' of patients regarding cognitive symptoms, in addition to psychological reasons, may have affected the cognitive function assessment. However, interestingly enough, 2 two of the studies that used both methods, subjective evaluation correlated with objective performance $(28,33)$.

Moreover, all studies including neuropsychological assessments are vulnerable to external validity limitations. These batteries may be both valid and reliable, but they do not reflect the responder's performance in the 'real world' and thus the use of such measurements decreases the generalizability of the findings. In other words, they burden a small effect size (53). In this review, this limitation was obvious in the study by Calvio et al (28), where the objective cognitive assessment failed to predict a decline in work output of patients, in contrast to the self-evaluated cognitive function instrument.

As far as the internal validity is concerned, according to the Newcastle-Ottawa Scale scoring, the ascertainment of exposure in patients and healthy individuals was not adequate in 6 out of 8 studies (26-28,30,31,33). Furthermore, only 3 have reported same method of exposure ascertainment in patients and a healthy population $(28,30,33)$. These findings indicate that there is a high risk of bias for the results of the majority of studies $(26,27,29-32)$.

Finally, there is also a concern regarding the use of cortisol levels to assess stress in 2 of the studies $(27,32)$. Previous data have yielded inconsistent results of a correlation between self-reported measures and stress biomarkers, including cortisol (54). Furthermore, a relevant study on patients with breast cancer assessing stress through the perceived stress scale and cortisol levels found no association between these measurements (55). Taken together, all the above are questioning the reliability of cortisol as a stress-related indicator in this patient group.

Hence, our findings are better interpreted as a general tendency to a correlation between stress and cognitive function in breast cancer patients, rather than a firm deduction.

In conclusion, this review supports that the cognitive dysfunction of patients with breast cancer may be partially stress-related, at least among the non-elderly population. Future research should investigate a potential effect of stress on the cognitive function of elderly patients, considering their increased risk for age-related cognitive disorders. Moreover, oncologists should be aware that even though chemotherapy has a considerable impact on the cognitive function of patients with breast cancer, this phenomenon may be partially stress-related, and therefore it should be addressed promptly.

\section{Acknowledgements}

Not applicable.

\section{Funding}

No funding was received.

\section{Availability of data and materials}

Data sharing is not applicable to this article, as no datasets were generated or analyzed during the current study.

\section{Authors' contributions}

All authors (AP, TS, ER, KK, AN, NA, CT, DAS and AK) contributed to the data analysis and the writing of the manuscript. AK was also involved in the conception of the study. AP, TS and AK were also involved in the literature search for this systematic review. All authors have read and approved the final manuscript.

\section{Ethics approval and consent to participate}

Not applicable.

\section{Patient consent for publication}

Not applicable.

\section{Competing interests}

DAS is the Editor-in-Chief for the journal, but had no personal involvement in the reviewing process, or any influence in terms of adjudicating on the final decision, for this article. The other authors declare that they have no competing interests.

\section{References}

1. Bray F, Ferlay J, Soerjomataram I, Siegel RL, Torre LA and Jemal A: Global cancer statistics GLOBOCAN estimates of incidence and mortality worldwide for 36 cancers in 185 countries. CA Cancer J Clin 68: 394-424, 2018.

2. Ferlay J, Steliarova-Foucher E, Lortet-Tieulent J, Rosso S, Coebergh JW, Comber H, Forman D and Bray F: Cancer incidence and mortality patterns in Europe: Estimates for 40 countries in 2012. Eur J Cancer 49: 1374-1403, 2013.

3. Jemal A, Ward E and Thun MJ: Recent trends in breast cancer incidence rates by age and tumor characteristics among U.S. women. Breast Cancer Res 9: R28, 2007.

4. Moore HC: An overview of chemotherapy-related cognitive dysfunction, or 'chemobrain'. Oncology (Williston Park) 28: 797-804, 2014.

5. Zheng Y, Luo J, Bao P, Cai H, Hong Z, Ding D, Jackson JC, Shu XO and Dai Q: Long-term cognitive function change among breast cancer survivors. Breast Cancer Res Treat 146: 599-609, 2014. 
6. Bompaire F, Durand T, Léger-Hardy I, Psimaras D and Ricard D Chemotherapy-related cognitive impairment or 'chemobrain': concept and state of art. Geriatr Psychol Neuropsychiatr Vieil 15: 89-98, 2017.

7. Heck JE, Albert SM, Franco R and Gorin SS: Patterns of dementia diagnosis in surveillance, epidemiology, and end results breast cancer survivors who use chemotherapy. J Am Geriatr Soc Sep 56: 1687-1692, 2008.

8. Jay TM, Rocher C, Hotte M, Naudon L, Gurden H and Spedding M: Plasticity at hippocampal to prefrontal cortex synapses is impaired by loss of dopamine and stress: Importance for psychiatric diseases. Neurotox Res 6: 233-244, 2004.

9. Pecori Giraldi F, Moro M and Cavagnini F; Study Group on the Hypothalamo-Pituitary-Adrenal Axis of the Italian Society of Endocrinology: Gender-related differences in the presentation and course of Cushing's disease. J Clin Endocrinol Metab 88: 1554-1558, 2003.

10. Tsolaki M: Post traumatic stress dementia: A new target for cholinesterase inhibitors. Neurobiol Aging 25: 26-27, 2004.

11. Boehmke MM and Dickerson SS: Symptom, symptom experiences, and symptom distress encountered by women with breast cancer undergoing current treatment modalities. Cancer Nurs 28: 382-389, 2005

12. Günüşen NP, İnan FŞ and Üstün B: Experiences of Turkish women with breast cancer during the treatment process and facilitating coping factors. Asian Pac J Cancer Prev 14: 3143-3149, 2013.

13. Hinnen C, Ranchor AV, Sanderman R, Snijders TA, Hagedoorn M and Coyne JC: Course of distress in breast cancer patients, their partners, and matched control couples. Ann Behav Med 36: 141-148, 2008.

14. Hoskins CN: Breast cancer treatment-related patterns in side effects, psychological distress, and perceived health status. Oncol Nurs Forum 24: 1575-1583, 1997.

15. Berhili S, Kadiri S, Bouziane A, Aissa A, Marnouche E, Ogandaga E, Echchikhi Y, Touil A, Loughlimi H, Lahdiri I, et al: Associated factors with psychological distress in Moroccan breast cancer patients: A cross-sectional study. Breast 31: 26-33, 2017.

16. Murman DL: The impact of age on cognition. Semin Hear 36 111-121, 2015.

17. Oh H, Mormino EC, Madison C, Hayenga A, Smiljic A and Jagust WJ: $\beta$-amyloid affects frontal and posterior brain networks in normal aging. Neuroimage 54: 1887-1895, 2011.

18. Dennis EL and Thompson PM: Functional brain connectivity using fMRI in aging and Alzheimer's disease. Neuropsychol Rev 24: 49-62, 2014

19. Wisdom NM, Mignogna $J$ and Collins RL: Variability in Wechsler Adult Intelligence Scale-IV subtest performance across age. Arch Clin Neuropsychol 27: 389-397, 2012.

20. Oosterman JM, Vogels RL, van Harten B, Gouw AA, Poggesi A, Scheltens P, Kessels RP and Scherder EJ: Assessing mental flexibility: Neuroanatomical and neuropsychological correlates of the Trail Making Test in elderly people. Clin Neuropsychol 24 203-219, 2010.

21. Park DC and Festini SB: Theories of memory and aging: A look at the past and a glimpse of the future. J Gerontol B Psychol Sci Soc Sci 72: 82-90, 2017.

22. Gosain R, Pollock Y and Jain D: Age-related disparity: Breast cancer in the elderly. Curr Oncol Rep 18: 69, 2016.

23. Sinha S, Panebianco L, Wu X, Wang D, Huang D and Sivapiragasam A: Abstract GS2-02: Efficacy and utilization trends of adjuvant chemotherapy for stage I, II, and III breast cancer in the elderly population: A National Cancer Database (NCDB) analysis. In: San Antonio Breast Cancer Symposium; San Antonio, 2018.

24. Moher D, Liberati A, Tetzlaff J, Altman DG and the PRISMA group: Preferred reporting items for systematic reviews and meta-analyses: The PRISMA statement. PLoS Medicine 6: e1000097, 2009.

25. Wells GA, Shea B, O'Connell D, Peterson J, Welch V, Losos M and Tugwell P: The Newcastle-Ottawa Scale (NOS) for assessing the quality of nonrandomised studies in metaanalyses. Ottawa Health Research Institute, Ottawa, 1999.

26. Menning S, de Ruiter MB, Kieffer JM, Agelink van Rentergem J, Veltman DJ, Fruijtier A, Oldenburg HS, Boven E, van der Meij S, Lustig V, et al: Cognitive impairment in a subset of breast cancer patients after systemic therapy-results from a longitudinal study. J Pain Symptom Manage 52: 560-569.e1, 2016.

27. Andreano JM, Waisman J, Donley L and Cahill L: Effects of breast cancer treatment on the hormonal and cognitive consequences of acute stress. Psychooncology 21: 1091-1098, 2012.
28. Calvio L, Peugeot M, Bruns GL, Todd BL and Feuerstein M: Measures of cognitive function and work in occupationally active breast cancer survivors. J Occup Environ Med 52: 219-227, 2010.

29. Debess J, Riis JØ, Pedersen L and Ewertz M: Cognitive function and quality of life after surgery for early breast cancer in North Jutland, Denmark. Acta Oncol 48: 532-540, 2009.

30. Levkovich I, Cohen M, Alon S, Kuchuk I, Nissenbaum B, Evron E, Pollack S and Fried G: Symptom cluster of emotional distress, fatigue and cognitive difficulties among young and older breast cancer survivors: The mediating role of subjective stress. J Geriatr Oncol 9: 469-475, 2018.

31. Henneghan A, Stuifbergen A, Becker H, Kesler S and King E: Modifiable correlates of perceived cognitive function in breast cancer survivors up to 10 years after chemotherapy completion. J Cancer Surviv 12: 224-233, 2018.

32. Kamen C, Scheiber C, Janelsins M, Jo B, Shen H and Palesh O: Effects of childhood trauma exposure and cortisol levels on cognitive functioning among breast cancer survivors. Child Abuse Negl 72: 163-171, 2017.

33. Hermelink K, Bühner M, Sckopke P, Neufeld F, Kaste J, Voigt V, Münzel K, Wuerstlein R, Ditsch N, Hellerhoff K, et al: Chemotherapy and post-traumatic stress in the causation of cognitive dysfunction in breast cancer patients. J Natl Cancer Inst: May 3, 2017 (Epub ahead of print). doi: https://doi.org/10.1093/jnci/ djx057.

34. Reyes-Gibby CC, Anderson KO, Morrow PK, Shete S and Hassan S: Depressive symptoms and health-related quality of life in breast cancer survivors. J Womens Health (Larchmt) 21: 311-318, 2012.

35. Ehlers DK, Aguiñaga S, Cosman J, Severson J, Kramer AF and McAuley E: The effects of physical activity and fatigue on cognitive performance in breast cancer survivors. Breast Cancer Res Treat 165: 699-707, 2017.

36. Yang H, Brand JS, Fang F, Chiesa F, Johansson AL, Hall P and Czene K: Time-dependent risk of depression, anxiety, and stress-related disorders in patients with invasive and in situ breast cancer. Int J Cancer 140: 841-852, 2017.

37. McDermott LM and Ebmeier KP: A meta-analysis of depression severity and cognitive function. J Affect Disord 119: 1-8, 2009.

38. Kim JI, Song Y, Lee JH, Kim HJ, Hong EJ, Kim SA, Jun YS and Chang OJ: Depression, stress and self-esteem according to treatment phase in patients with breast cancer. Indian J Sci Technol 8: 1-7, 2015.

39. Franzen MD (ed): Practical and methodological considerations regarding reliability. In: Reliability and Validity in Neuropsychological Assessment. Springer Science and Business Media, New York, NY, pp15-26, 2013.

40. Fan HG, Houédé-Tchen N, Yi QL, Chemerynsky I, Downie FP, Sabate K and Tannock IF: Fatigue, menopausal symptoms, and cognitive function in women after adjuvant chemotherapy for breast cancer: 1- and 2-year follow-up of a prospective controlled study. J Clin Oncol 23: 8025-8032, 2005.

41. Schilder CM, Seynaeve C, Beex LV, Boogerd W, Linn SC, Gundy CM, Huizenga HM, Nortier JW, van de Velde CJ, van Dam FS, et al: Effects of tamoxifen and exemestane on cognitive functioning of postmenopausal patients with breast cancer: Results from the neuropsychological side study of the tamoxifen and exemestane adjuvant multinational trial. J Clin Oncol 28: 1294-1300, 2010

42. Silverman DH, Dy CJ, Castellon SA, Lai J, Pio BS, Abraham L, Waddell K, Petersen L, Phelps ME and Ganz PA: Altered frontocortical, cerebellar, and basal ganglia activity in adjuvant-treated breast cancer survivors 5-10 years after chemotherapy. Breast Cancer Res Treat 103: 303-311, 2007.

43. de Ruiter MB, Reneman L, Boogerd W, Veltman DJ, Caan M, Douaud G, Lavini C, Linn SC, Boven E, van Dam FS, et al: Late effects of high-dose adjuvant chemotherapy on white and gray matter in breast cancer survivors: Converging results from multimodal magnetic resonance imaging. Hum Brain Mapp 33: 2971-2983, 2012.

44. Pelekasis P, Matsouka I and Koumarianou A: Progressive muscle relaxation as a supportive intervention for cancer patients undergoing chemotherapy: A systematic review. Palliat Support Care 15: 465-473, 2017.

45. Pelekasis P, Zisi G, Koumarianou A, Marioli A, Chrousos G, Syrigos K and Darviri C: Forming a stress management and health promotion program for women undergoing chemotherapy for breast cancer: A pilot randomized controlled trial. Integr Cancer Ther 15: 165-174, 2016.

46. Maizes V, Rakel D and Niemiec C: Integrative medicine and patient-centered care. Explore (NY) 5: 277-289, 2009. 
47. Cimprich B, So H, Ronis DL and Trask C: Pre-treatment factors related to cognitive functioning in women newly diagnosed with breast cancer. Psychooncology 14: 70-78, 2005.

48. Wefel JS, Lenzi R, Theriault R, Buzdar AU, Cruickshank S and Meyers CA: 'Chemobrain' in breast carcinoma?: A prologue. Cancer 101: 466-475, 2004.

49. Hermelink K, Untch M, Lux MP, Kreienberg R, Beck T, Bauerfeind I and Münzel K: Cognitive function during neoadjuvant chemotherapy for breast cancer: Results of a prospective, multicenter, longitudinal study. Cancer 109: 1905-1913, 2007.

50. Schagen SB, Muller MJ, Boogerd W, Mellenbergh GJ, van Dam FS Change in cognitive function after chemotherapy: a prospective longitudinal study in breast cancer patients. J Natl Cancer Inst 98 : 1742-5, 2006

51. Stewart A, Bielajew C, Collins B, Parkinson M and Tomiak E: A meta-analysis of the neuropsychological effects of adjuvant chemotherapy treatment in women treated for breast cancer. Clin Neuropsychol 20: 76-89, 2006.

52. Héry C, Ferlay J, Boniol M and Autier P: Quantification of changes in breast cancer incidence and mortality since 1990 in 35 countries with Caucasian-majority populations. Ann Oncol 19: 1187-1194, 2008.
53. Franzen MD (ed): Validity as applied to neuropsychological assessment. In: Reliability and Validity in Neuropsychological Assessment. Springer Science and Business Media, New York, NY, pp33-54, 2013.

54. Hellhammer DH, Wüst S and Kudielka BM: Salivary cortisol as a biomarker in stress research. Psychoneuroendocrinology 34 : 163-171, 2009.

55. Wu SM, Yang HC, Thayer JF and Andersen BL: Association of the physiological stress response with depressive symptoms in patients with breast cancer. Psychosom Med 76: 252-256, 2014.

(i) (9) This work is licensed under a Creative Commons Attribution-NonCommercial-NoDerivatives 4.0 International (CC BY-NC-ND 4.0) License. 